\title{
UV FLUXES IN ELLIPTICAL GALAXIES
}

\author{
CESARE CHIOSI \\ Astronomy Department, University of Padua \\ Vicolo Osservatorio 5, 35122 Padua, Italy
}

\begin{abstract}
.
The key properties of elliptical galaxies are compared to present-day models calling attention on several points of contradiction. The nature of the UV fluxes and their usefulness to cast light on the age-metallicity dilemma are discussed. Finally, preliminary results of an attempt aimed at reconciling theory with observations by means of an new IMF are presented.
\end{abstract}

\section{Introduction}

Understanding the nature of the UV excess in the spectrum of normal elliptical galaxies is tightly related to the past history of star formation and chemical enrichment, and in turn to the mechanism of galaxy formation itself. On these grounds, the origin of the UV fluxes cannot be searched independently from other key properties of elliptical galaxies. In the following we call attention on several points of controversy and attempt to derive a coherent explanation in which the UV fluxes and a new initial mass function (IMF) will play an important role.

\section{UV fluxes in elliptical galaxies: the data}

According to the pioneering study of Burstein et al. (1988): (1) All studied elliptical galaxies have detectable UV flux short-ward of about $2000 \AA$. (2) There are large galaxy to galaxy differences in the level of the UV flux. The intensity of the UV emission is measured by the colour $(1550-\mathrm{V})$. (3) The colour $(1550-\mathrm{V})$ correlates with the index $\mathrm{Mg} 2$, the velocity dispersion $\Sigma$, and the luminosity (mass) of the galaxy. The few galaxies (e.g. NGC 205) in which active star formation is seen do not obey these relations. (4) An important constraint is posed by the HUT observations by Ferguson 


\section{UV FLUXES}

et al. (1991) and Ferguson \& Davidsen (1993) of the UV excess in the bulge of M31. In this case the UV emission shows a drop-off short-ward of about $1000 \AA$ whose interpretation requires that the temperature of the emitting source must be about $25,000 \mathrm{~K}$. Only a small percentage of the $912 \leq \lambda \leq 1200 \AA$ flux can be coming from stars hotter than $30,000 \mathrm{~K}$ and cooler than $20,000 \mathrm{~K}$.

\section{The analog of the G-Dwarf problem}

Analyzing the integrated spectral energy distributions (ISED) of prototype galaxies with different intensity of the UV flux, Bressan et al. (1994, BCF) first noticed that the adoption of the closed-box model to follow the chemical history of these systems inevitably leads to an a excess of flux in the region $2000 \AA$ to $3500 \AA$, caused by the excess of low metallicity stars implicit in this type of model. In perfect analogy with the G-dwarf problem in the solar vicinity (cf. Tinsley 1980), whose solution was found in models with infall (Lynden-Bell 1975), Tantalo et al. (1996, TCBF) showed that also in the case of elliptical galaxies the infall scheme predicts very few stars of low metallicity thus removing the above discrepancy. This analysis clearly shows how much the past history of chemical enrichment and star formation in turn bear on key properties of elliptical galaxies and cast light on the kind of metallicity distribution (number of stars per metallicity bin) that ought to exist in these galaxies.

\section{Hints from chemical abundances}

Direct determinations of metallicities in elliptical galaxies are still rather limited. Most of the information comes from metal line-strength indices (Carollo et al. 1993, Carollo \& Danziger 1994, Davies et al. 1993) and broad-band colours (Schombert et al. 1993) together with their spatial gradients. The evidence arises that the metallicities for elliptical galaxies are solar or larger. Whether the metallicity distribution have tails extending to very low and high metallicities is by far more controversial. Limited to the case of the Galactic Bulge, McWilliam \& Rich (1994) show from spectroscopic studies that the mean metallicity is slightly lower than solar and long tails towards the metal-poor are present; Bertelli et al. (1995) analyzing the CMD of the region Terzan $1(l=357.5 ; b=1.0)$ argue that old stars with a large range of metallicity $(0.008<\mathrm{Z}<0.08)$ should exist. Furthermore, for bright elliptical galaxies there are also indications that the $\alpha$-elements $(\mathrm{O}, \mathrm{Mg}, \mathrm{Si}$, etc..) are enhanced with respect to Fe. Although passing from narrow band indices to chemical abundances is not a straight process (age and low metallicity effects could be present, cf. Bressan et al. $1997 \mathrm{a})$, arguments are given to conclude that the average $[\mathrm{Mg} / \mathrm{Fe}]$, in par- 


\section{Chiosi}

ticular, exceeds that of the most metal rich stars in the solar vicinity by about $0.2-0.3 \mathrm{dex}$, and the ratio $[\mathrm{Mg} / \mathrm{Fe}]$ increases with the galactic mass up to the this value (cf. Matteucci 1994). The enhancement of $\alpha$-elements is particularly stringent as the supersolar value for giant ellipticals hints that an unique source of nucleosynthesis has been contributing to chemical enrichment. Natural candidates are the massive stars exploding as type II $\mathrm{SN}$ and thus releasing elemental species ( $\mathrm{Mg}$ and $\mathrm{Fe}$ in particular) in fixed proportions. As $\mathrm{Mg}$ is known to be produced only by massive stars, the decrease of $[\mathrm{Mg} / \mathrm{Fe}]$ with the galactic mass implies another source of Fe. Type I SNs from mass accreting White Dwarfs in binary systems are the most obvious explanation. Finally, the metallicity distribution (together with the age) will drive the dominant source of UV radiation (see below), whereas the degree of enhancement in $\alpha$-elements will perhaps measure the overall duration of bulk activity of star formation: short in massive galaxies and long in the low mass ones.

\section{The color-magnitude relation}

Elliptical galaxies obey a mean color-magnitude relation (CMR): colors get redder at increasing luminosity, cf. Bower et al. (1992) for galaxies in the Virgo and Coma clusters and Schweizer \& Seitzer (1992) for galaxies in small groups and field. The cluster CMR is particularly narrow, whereas the field CMR is more disperse. Long ago Larson (1974) postulated that the present-day CMR is the consequence of SN driven galactic winds. With the standard star formation scenario, first massive galaxies get higher mean metallicities, second because of their stronger gravitational potential eject at later epochs their gaseous content heated up by SN explosions than the less massive ones. Since then, many theoretical models of various complexity have matched the slope of the CMR. As far as the width of the CMR is concerned, it has been argued that most of cluster galaxies are nearly coeval with age ranging from 13 to 15 Gyr (Bower et al. 1992), whereas field galaxies are perhaps compatible with recent mergers spread over several Gyr (Schweizer \& Seitzer 1992). How much would other properties of elliptical galaxies (UV colours and/or narrow band indices, in particular) reflect the different history of star formation in the two alternatives ? Is this interpretation compatible with the hints from chemical properties?

\section{The fundamental plane}

Elliptical galaxies do not populate uniformly the parameter space with coordinates the central velocity dispersion $\Sigma$, effective radius $R_{e}$, and surface brightness $I_{e}$. They cluster around a plane called the Fundamental Plane. Using the coordinate system defined by Bender et al. (1992), the physi- 
cal coordinates translated to $k_{1} \propto \log \left[a_{1} M\right], k_{2} \propto \log \left[a_{2}(M / L) I e^{3}\right]$ and $k_{3} \propto \log \left[a_{3}(M / L)\right]$, with $a_{1}, a_{2}$, and $a_{3}$ suitable constants. Of particular relevance is the projection of the FP onto the $k_{1}-k_{3}$ plane, where the FP is seen edge on. Limited to the case of the Virgo elliptical galaxies to avoid distance uncertainties, the relation $k_{3}=0.15 k_{1}+0.36$ with $\sigma\left(k_{3}\right)=0.05$ is found to hold (cf. Ciotti et al. 1996 for details). The ratio $(M / L)$ increases with the galaxy mass (tilt of the FP). The tilt and tightness of the FP have so far eluded simple explanations (cf. Renzini \& Ciotti 1993, Ciotti et al. 1996).

\section{Sources of UV fluxes}

Most likely, the UV excess owes its origin to an old component that gets hot enough to power the integrated spectral energy distribution (ISED) of a galaxy in the far UV regions. Several possible candidates are envisaged (cf. Greggio \& Renzini 1990, BCF and TCBF). The appearance of the various types of UV sources is governed by several important physical factors, each of which is affected by a certain degree of uncertainty still far from being fully assessed. These are the efficiency of mass loss during the RGB and AGB phases, the enrichment law $\Delta \mathrm{Y} / \Delta \mathrm{Z}$, and finally for the specific case of P-AGB stars the detailed relation between the initial and final mass of the stars at the end of the AGB phase.

(1) The classical post asymptotic giant branch (P-AGB) stars (see Bruzual \& Charlot 1993, Charlot \& Bruzual 1991), which are always present in the stellar mix of a galaxy. However, they cannot be the sole source of UV flux because of their high mean temperature (about 100,000 K) and lack of sufficient fuel (cf. Greggio \& Renzini 1990). Another point of uncertainty is the precise relation between the P-AGB mass and the turn-off mass (and hence age), which is far from being established.

(2) Very blue HB (VB-HB) stars of extremely low metallicity (Lee 1994). These stars have effective temperatures hotter than about $15,000 \mathrm{~K}$ but much cooler than those of the P-AGB stars. Therefore, depending on their actual $\mathrm{T}_{\text {eff }}$, they can generate ISEDs in agreement with the observational data provided that the age is let vary from galaxy to galaxy (Park \& Lee 1997). It must be checked, however, whether the presence of low metallicity stars is compatible with the observed ISED in the range $2000<\lambda<3500$ $\AA$ (G-Dwarf problem).

(3) The H-HB and AGB-manqué stars of high metallicity (say $\mathrm{Z}>0.07$ ) which are expected to be present albeit in small percentages in the stellar content of bulges and elliptical galaxies in general. Indeed, these stars have effective temperatures in the right interval and generate ISEDs whose intensity drops short-ward of about $1000 \AA$ by the amount indicated by the 


\section{Chiosi}
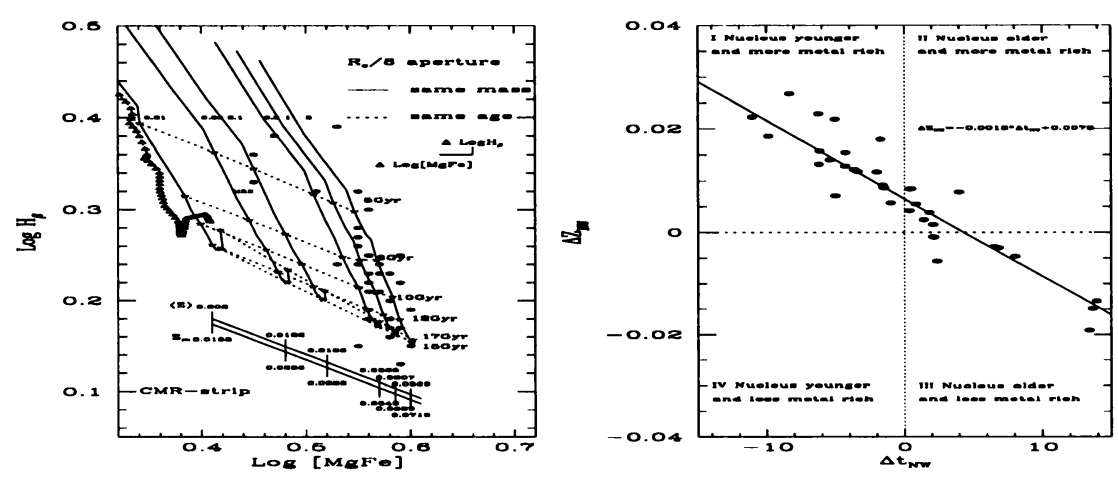

Figure 1. Left : The $\mathrm{H}_{\beta}-[\mathrm{MgFe}]$ plane of theoretical models and observational data. Right : the relation between $\Delta t_{W N}$ and $\Delta Z_{W N}$, i.e. the age and metallicity difference between the whole galaxy and its central regions

observational data. With normal mass loss and $\Delta \mathrm{Y} / \Delta \mathrm{Z}$, the first $\mathrm{H}-\mathrm{HB}$ and AGB manque stars occurs at the age of about $5.6 \mathrm{Gyr}$. This age is lowered if $\Delta \mathrm{Y} / \Delta \mathrm{Z}$ is higher than 2.5 (cf. Pagel et al. 1992 ).

(4) Finally, the analog of the above H-HB and AGB-manque stars, but generated by enhancing the mass loss rate during the RGB phase at increasing metallicity. These type of stars have been named by Dorman et al. $(1993,1995)$ extremely hot HB objects (E-HB). They share nearly the same properties of the H-HB and AGB-manque stars. The main difficulty with this option is the uncertainty concerning the metallicity dependence of the mass loss rate (cf. Carraro et al. 1996).

\section{The age-metallicity dilemma}

Metallicity and age concur to determine the mean properties of elliptical galaxies. Unfortunately, both act in the same sense, i.e. the stellar content of a galaxy gets redder at increasing age and metallicity thus giving rise to the well known age-metallicity dilemma. The indices $\mathrm{H}_{\beta}$ and $[\mathrm{MgFe}]$ are particularly suited to investigate this problem, because $\mathrm{H}_{\beta}$ is a measure of the turn-off colour and luminosity, and age in turn, whereas $[\mathrm{MgFe}]$ is more sensitive to the RGB colour and hence metallicity. Gonzales (1993) analyzing the distribution of a sample of galaxies in the $\mathrm{H}_{\beta}-[\mathrm{MgFe}]$ plane of single stellar populations with different metallicity and age concluded that a large spread in age seems to exist. Bressan et al. (1996, BCT), implementing the calibration of Worthey et al. (1994) for the line strength indices into the models of BCF and TCBF and adding two more dimensions to the problem, i.e. the intensity of the UV excess and the velocity dispersion $\Sigma$, tackled the question whether or not these systems span a large age 


\section{UV FLUXES}

range. The models were compared with the Gonzales(1993) data for the nuclear region $(R e / 8)$ as shown by the left panel of Fig. 1 . The solid lines are loci of constant mass (in units of $10^{12} \mathrm{M}_{\odot}$ ) and nearly constant mean and maximum metallicities, whereas the dotted lines are loci of constant age (in Gyr). Finally, the locus named CMR-strip is the analog of the the CMR on the notion that this is a mass-metallicity sequence of old $(13 \div 15$ Gyr), nearly coeval objects. An arbitrary shift along the $\mathrm{H}_{\beta}$-axis has been applied for the sake of clarity. The following conclusions were reached (1) In spite of their different luminosity and mass, most galaxies seem to possess nearly identical chemical structures; (2) Galaxies do not distribute along the locus expected from the CMR of coeval old objects. In contrast, they seem to follow a sequence of about constant metallicity and varying age. (3) The distribution of galaxies in the $\mathrm{H}_{\beta}-[\mathrm{MgFe}]$ plane is neither a sequence of sole metallicity with bluer galaxies significantly more metal-poor than the red ones, nor a sequence of sole age with bluer galaxies significantly younger than the red ones. One may argue that the large spread along the $\mathrm{H}_{\beta}$ direction could be the result of a recent episode of star formation (burst) shifting the galaxies from their natural location (the CMR-strip) to the one we actually see. The major drawback with this idea is that a sort of synchronization is required. Using the simultaneous inspection of the data in the $\mathrm{H}_{\beta}-[\mathrm{MgFe}]-(1550-\mathrm{V})-\Sigma$ space, BCT suggested that the explanation is more intrigued than the simple burst hypothesis. To this aim, BCT examined the information provided by the difference in $\mathrm{H}_{\beta}$ and $[\mathrm{MgFe}]$ observed within two different aperture, i.e. Re/8 (nuclear region: $\mathrm{N}$ ) and $R e / 2$ (whole galaxy: W). The differences are indicated by $\Delta \mathrm{H}_{\beta_{N W}}$ and $\Delta[\mathrm{MgFe}]_{N W}$. Translating $\Delta \mathrm{H}_{\beta_{N W}}$ and $\Delta[\mathrm{MgFe}]_{N W}$ into $\Delta t_{N W}$ and $\Delta Z_{N W}$, i.e. in the age and metallicity difference between the nucleus and the whole galaxy, respectively, BCT get the correlation shown in the right panel of Fig. 1. Of the four quadrant's, the one characterized by $\Delta t_{N W}<0$ and $\Delta Z_{N W}>0$ is particularly rich of galaxies. In this region, the nucleus is younger and more metal-rich than the external regions of the galaxy. This corresponds to a sort of out-inward process of galaxy and star formation that in some cases continued for significantly long periods of time. Whether the star formation process took place continuously or in a series of episodes cannot be said. The age difference is found to be inversely proportional to the velocity dispersion $\Sigma$ (perhaps mass).

\section{How to reconcile things?}

The scenario emerging from the analysis of the gradients in $\mathrm{H}_{\beta}$ and $[\mathrm{MgFe}]$, the current data on narrow band indices (Carollo et al. 1993, Carollo \& Danziger 1994) together with the expected trend for the ratio $[\mathrm{Mg} / \mathrm{Fe}]$ 


\section{Chiosi}

with galaxy mass (Davies et al. 1993; Matteucci 1994), suggest that the duration of the star formation activity was shorter in massive galaxies than in the low mass ones. This is exactly the opposite of what is implied by the galactic wind mechanism required to explain the slope of the CMR. In addition to this, we have the embarrassing result that current models of elliptical galaxies that fairly match many key properties of these systems are still unable to account for the tilt of the FP. As a matter of fact, the $M / L_{B}$ ratios predicted by the models run almost flat at increasing luminosity (mass) of the galaxy over a wide range of ages (cf. the $M / L_{B}$ versus $L_{B}$ relations of the $\mathrm{BCT}$ and TCBF models). It goes without saying that at given mass, the $M / L_{B}$ ratios increase with the age. Unless faint, low mass galaxies are truly young objects (in the sense that dominant star formation started much later than in the massive ones), no viable solution can be found without relaxing one of the key assumptions. Among the various suggestions advanced to explain the FP tilt, Renzini \& Ciotti (1993) explored the effect of varying the IMF and concluded that a major but suitable change of the IMF is required so that the tightness of the FP is maintained. Padoan et al. (1996) have recently proposed a new type of IMF that may turn out to be able to reconcile the above points of difficulty.

\section{An universal IMF}

Padoan's et al. (1996) IMF stems from a statistical description of the density field emerging from randomly forced supersonic flows in star forming regions. The hydrodynamical simulations of supersonic flows shows that very large density contrasts can develop with log-normal probability distribution. The resulting IMF is

$$
\int_{0}^{\infty} \phi(M) d M=\int_{0}^{\infty} \frac{2 B^{2}}{\left(2 \pi \sigma^{2}\right)^{0.5}} M^{-3} \exp \left[-0.5\left(\frac{2 \ln M-A}{\sigma}\right)^{2}\right] d M=1
$$

The quantities $A, B$ and $\sigma$ are defined by the following relations: $A=$ $2 \ln B+0.5 \sigma^{2}, B=1.2 \times(T / 10)^{1.5} \times(n / 1000)^{-0.5}, \sigma^{2}=\ln \left[1+0.36\left(M a^{2}-1\right)\right]$, in which $\sigma$ is the standard deviation of the number density distribution in the field with respect to the mean. $M a^{2}$ is the Mach number given by $M^{2}=\left(\Sigma / v_{s}\right)^{2}, T$ is the temperature in $\mathrm{K}, n$ is the number density in $\mathrm{cm}^{-1}, v_{s}$ the sound velocity, and $\Sigma$ the velocity dispersion of the gas (in $\mathrm{km} / \mathrm{s}$ ). This IMf has long tail at high masses, an exponential cutoff at the smallest masses, and a characteristic peak mass

$$
M_{\text {max }} \simeq 0.2 M_{\odot} \times(T / 10)^{2} \times(n / 1000)^{-0.5} \times(\Sigma / 2.5)^{-1}
$$



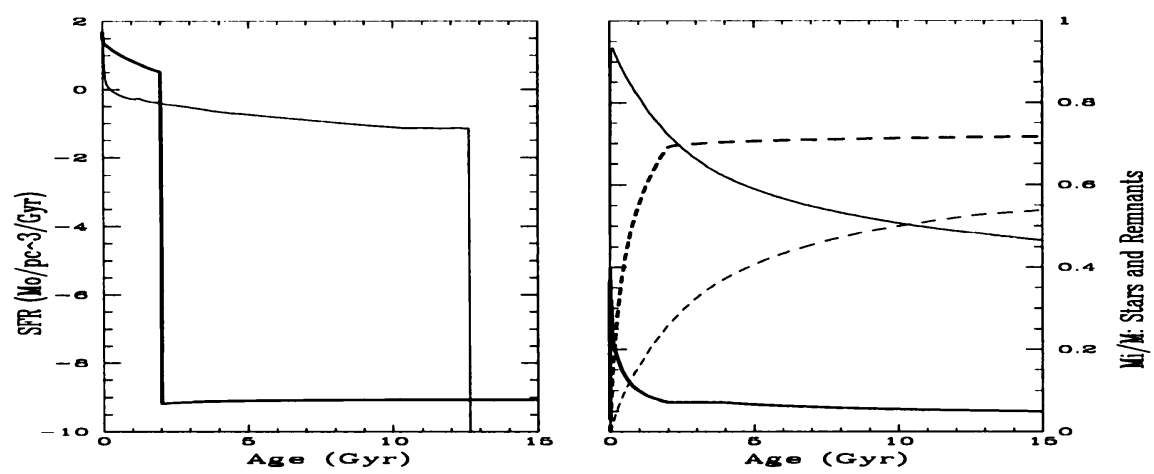

Figure 2. Left : Rate of star formation (in $\mathrm{M}_{\odot} / \mathrm{pc}^{3} / \mathrm{Gyr}$ ) as a function of the age in the nuclear region $(r / R e=0.1)$ of the $3 \times 10^{12} \mathrm{M}_{\odot}$ (heavy lines) and $1 \times 10^{9} \mathrm{M}_{\odot}$ (thin lines) galaxies. The efficiency of star formation is $\nu=50$. Right : The fractionary mass of visible stars (solid lines) and remnants (dashed lines) as a function of the age in Gyr for the models in the right panel

and a slope continuously varying with the mass. The IMF gets flatter and $M_{\max }$ gets higher at increasing temperature or decreasing density and velocity dispersion. The opposite occurs reversing the variations of the three physical parameters. See Padoan et al. (1996) for all other details. Chiosi et al. (1997) and Bressan et al. (1997b) have implemented the new IMF in the models of elliptical galaxies of Tantalo et al. (1997) in which spatial gradients of density and star formation are taken into account. The key assumptions and results are the following:

(1) Elliptical galaxies are modelled as a spherical distribution of luminous and dark matter, in which the mass of the luminous material is imposed to obey the present-day density profile inferred from the $R^{1 / 4}$ law (Young 1976). The density of gas $\rho(r, t)_{g}$ (and stars in turn) is therefore a function of time and radial distance from the center. For the sake of simplicity, the models below adopt the closed box scheme. The rate of star formation is $\dot{\rho}(r, t)=\nu(r, t) \rho(r, t)_{g}$, where $\nu(r, t)$ is a suitable efficiency parameter. The results presented here refer to $\nu=50$.

(2) The IMF is calculated assuming for $\Sigma$ the virial velocity dispersion, for the gas density its current value $\rho(r, t)_{g}$, and deriving the temperature $T$ from the energy equation $\dot{E}=H-\Lambda$, where $H$ and $\Lambda$ are the heating and cooling rate, respectively. Heating is due to the energy deposit from SN explosions and stellar winds from stars, whereas cooling is regulated by radiative processes (no details are given here for the sake of brevity). Therefore, the IMF continuously varies with time, radial distance, and galaxy mass.

(3) Passing from a low to a high mass galaxy, the mean density decreases (a suitable mass-radius relation is implied). The same is true going from the 


\section{Chiosi}

center to the periphery of a galaxy of given mass. At given velocity dispersion, the change in density drives the IMF which is more and more skewed towards higher masses. The relative number of SN increases, the energy deposit gets higher, the gas temperature increases, but owing to the more efficient production of metals radiative cooling becomes more efficient too. This complex game among the various factors establishes a self-regulating mechanism by which the gas gets hotter in the low density regions than in the high density ones. The same is true going from a low mass (high mean density) galaxy to a high mass (low mean density) one. The resulting behaviour of the IMF automatically secures that very few low mass stars are produced thus solving the G-Dwarf problem and rendering the infall scheme superfluous. Finally, at proceeding evolution, the gas energy eventually exceeds the gravitational energy and galactic winds start, earlier in massive than in low mass galaxies.

(4) As a result of all this, in a galaxy of given mass the central regions form stars for much longer periods of time than the external ones: galaxies are built up by an out-in process. Two cases are shown in the left panel of Fig. 2, displaying the rate of star formation (in units of $\mathrm{M}_{\odot} / \mathrm{pc}^{3} / \mathrm{Gyr}$ ) as a function of the age limited to the central region $(r / R e=0.1)$ in galaxies of different total mass. The right panel shows the fractionary mass in visible stars (solid lines) and remnants (dashed lines) as function of the age. Note the much earlier onset of galactic winds in massive galaxies.

(5) As far as photometric properties and $M / L_{B}$ ratios are concerned, the results are the following: at the age of $15 \mathrm{Gyr}$, the nuclear region of the $1 \times 10^{9} \mathrm{M}_{\odot}$ galaxy has $(B-V)=0.98,(V-K)=2.93, M_{V}=-16.04$ and $M / L_{B}=5.5$, whereas the $3 \times 10^{12} \mathrm{M}_{\odot}$ galaxy has $(B-V)=1.12$, $(V-K)=3.35, M_{V}=-22.34$. and $M / L_{B}=48$ (upper limit). Taken at face values, these numbers already account for the tilt of the FP. The agreement will be certainly improved taking into account the contribution from all other regions not included here. Finally, the $M / L_{B}$ ratio is expected to increase from the center to the periphery, a well known observational result.

(6) On the average, owing to the different duration of the star forming activity and skewness of the IMF towards the high mass end, massive galaxies are more enhanced in $\alpha$-elements than the low mass ones. We get mean $[\mathrm{Mg} / \mathrm{Fe}]=0.3$ and $[\mathrm{Mg} / \mathrm{Fe}]=0$ for the $3 \times 10^{12} \mathrm{M}_{\odot}$ and $1 \times 10^{9} \mathrm{M}_{\odot}$ galaxy, respectively. Conversely, inside a galaxy the enhancement in $\alpha$-elements is expected to be higher at the periphery than at the center. This apparently opposes to the observational gradients in the line strength index $M g_{2}$. However, recalling that the longer period of stellar activity at the center implies much higher metallicities, whether or not the trend in chemical elements contradicts the hint from line strength indices cannot be assessed without a detailed calculation of these for a mix of stars with different ages and 


\section{UV FLUXES}

metallicities.

(7) Finally, despite the shorter duration of the star forming activity and earlier winds, the mean metallicity increases with the galaxy mass owing to the compensatory effect caused by the shift of the IMF towards higher stellar masses. The classical interpretation of the CMR is still valid as indicated by the colors and magnitudes quoted above.

Study financially supported by the italian MURST and Space Agency.

\section{References}

Bender, R., Burstein, D., \& Faber, S.M., 1992, ApJ, 399, 462

Bertelli, G., Bressan, A., Chiosi, C., Ng, Y.K., \& Ortolani, S., 1995, A\&A, 301, 381

Bower, R.G., Lucey, J.R., \& Ellis, R.S., 1992, MNRAS, 254, 601

Bressan, A., Chiosi, C., \& Fagotto, F., 1994, ApJS, 94, 63, BCF

Bressan, A., Chiosi, C., \& Tantalo, R., 1996, A\&A, 311, 425, BCT

Bressan, A., Tantalo R., Chiosi C., \& Portinari, L., 1997a, A\&A, to be submitted

Bressan, A., Chiosi, C., Tantalo, R., \& Portinari, L., 1997b, A\&A, to be submitted

Bruzual, G., \& Charlot, S., 1993, ApJ, 405, 538

Burstein, D., Bertola, F., Buson, L.M., Faber, S.M., \& Lauer, T.R., 1988, ApJ, 328440

Carollo, C. M., \& Danziger, I.J., 1994, MNRAS, 270, 523

Carollo, C.M., Danziger, I.J., \& Buson, L., 1993, MNRAS, 265, 553

Carraro, G., Girardi, L., Bressan, A., \& Chiosi, C., 1996, A\&A, 305, 849

Charlot, S., \& Bruzual, G., 1991, ApJ, 367, 126

Chiosi, C, Bressan, A., \& Portinari, L., Tantalo, R., 1997, ApJ, to be submitted

Ciotti, L., Lanzoni, B., \& Renzini, A., 1996, MNRAS 282, 11996

Davies, R. L., Sadler, E. M., \& Peletier, R.F., 1993, MNRAS, 262, 650

Dorman, B., O'Connell, R.W., \& Rood, R.T., 1995, A\&A, 442, 105

Dorman, B., Rood, R.T., \& O'Connell, R.W., 1993, A\&A, 419, 516

Ferguson, H.C., \& Davidsen, A.F., 1993, ApJ, 408, 92

Ferguson, H.C., Davidsen A.F., Kriss, G.A., et al., 1991, ApJ, 382, L69

Gonzales, J.J., 1993, Ph.D. Thesis, Univ. California, Santa Cruz

Greggio, L., \& Renzini, A., 1990, ApJ, 364, 35

Larson, R.B., 1974, MNRAS, 166, 585

Lee, Y-W., 1994, ApJ, 423, 248

Lynden-Bell, D., 1975, Vistas in Astronomy, 19, 299

Matteucci, F., 1994, A\&A, 288, 57

McWilliam, A., \& Rich, R.M., 1994, ApJS, 91, 749

Padoan, P., Nordlund, A.P., \& Jones, B.J.T., 1996, preprint

Park, J.-H., \& Lee, Y.-K., 1977, ApJ, 476, in press

Pagel, B.E.J., Simonson, E.A., Terlevich, R.J., \& Edmunds, M.G., 1992, MNRAS, 255, 325

Renzini, A., \& Ciotti, L., 1993, ApJ, 416, L49

Schombert, J.M., Halan, P.C., Barsony, M., \& Rakos, K.D., 1993, AJ, 106, 923

Schweizer, F., \& Seitzer, P., 1992, AJ, 104, 1039

Tantalo, R., Chiosi, C., Bressan, A., \& Fagotto, F., 1996, A\&A, 311, 361, TCBF

Tantalo, R., Bressan, A., Chiosi, C., \& Portinari, L., 1997, A\&A, to be submitted

Tinsley, B.M., 1980, Fundamentals of Cosmic Physics, 5, 287

Young, P.J., 1976, AJ, 81, 807

Worthey, G., Faber, S.M., Gonzales, J.J., \& Burstein, D., 1994, ApJS, 94, 687 